\title{
Exemple de protection du littoral : Reconquête de la plage de Saint-Aygulf (département du Var)
}

\author{
M. Chavand \\ Chef de Subdivision Maritime-D.D.E. Var \\ C. Migniot \\ Docteur es-Sciences - Ingénieur Conseil
}

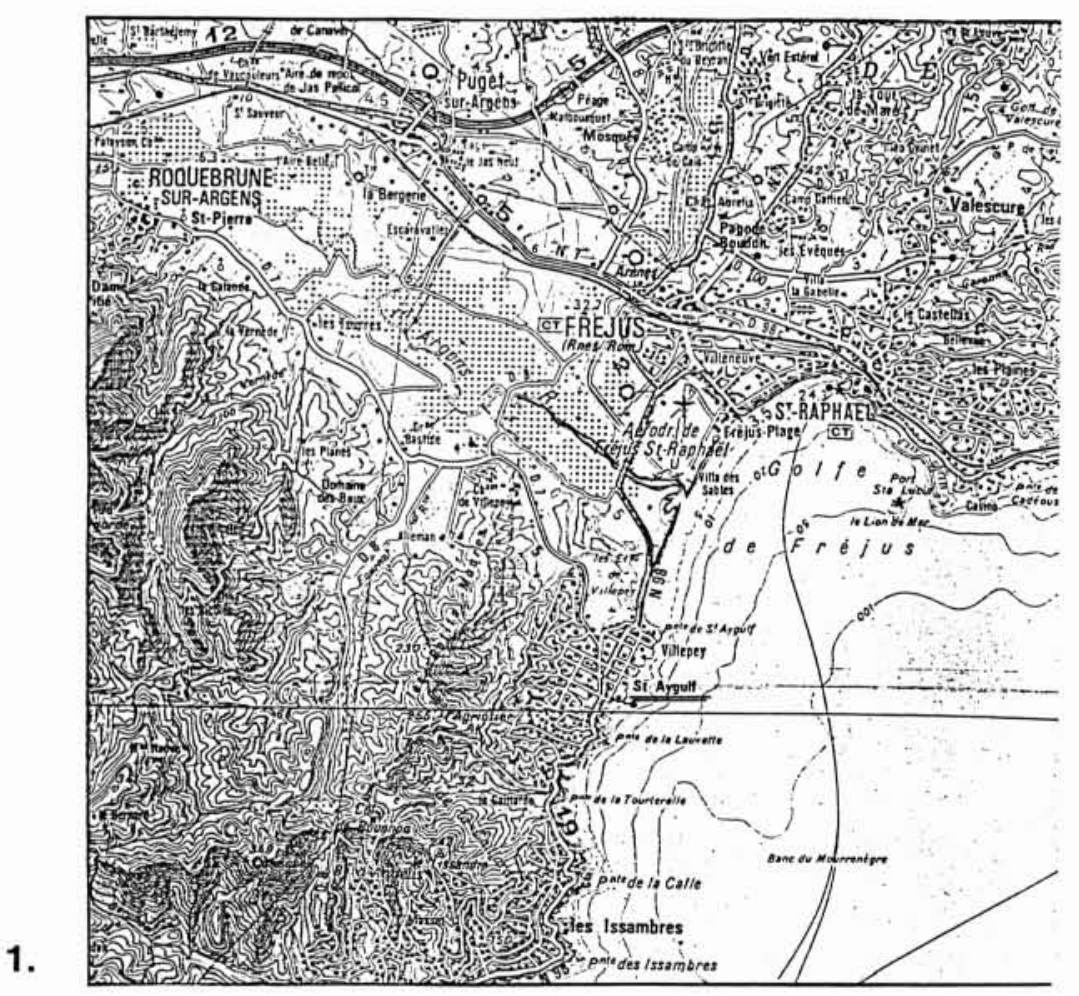

Situation: La plage de Saint-Aygulf est située au SudOuest du Golfe de Fréjus le long d'un littoral très exposé aux houles d'Est et Sud-Est, qui s'étend sur 5 kilomètres de la ville de Fréjus à la pointe de Saint-Aygulf (fig. I).
Cette plage a été soumise, au cours des denières décennies, à une dégradation très importante qui a entraîné une diminution de sa capacité d'accueil balnéaire nécessitant d'entreprendre des travaux de protection et de reconquête. 


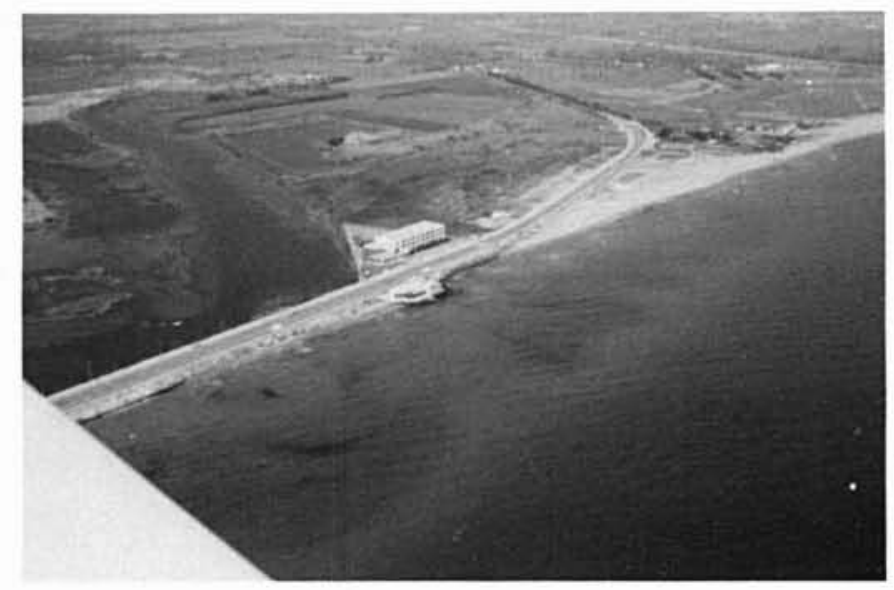

2. La plage dégradée en 1980.

3. Le littoral après la rupture du barrage de Malpasset.

4. Embouchure de l'Argens.

5. Vue générale du modèle réduit.

6. Evolution de l'embouchure de l'Argens pendant les extractions.

7. Reformation de la plage d'embouchure après l'arrêt des extractions.

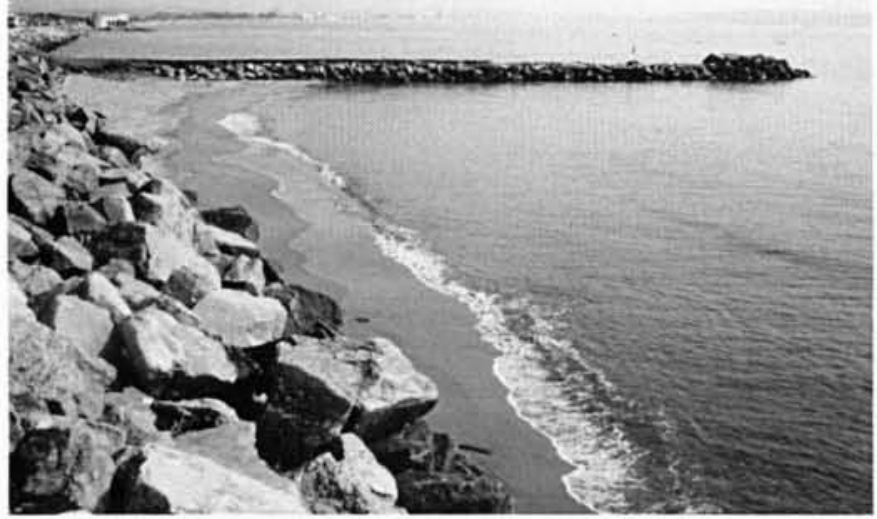

2.

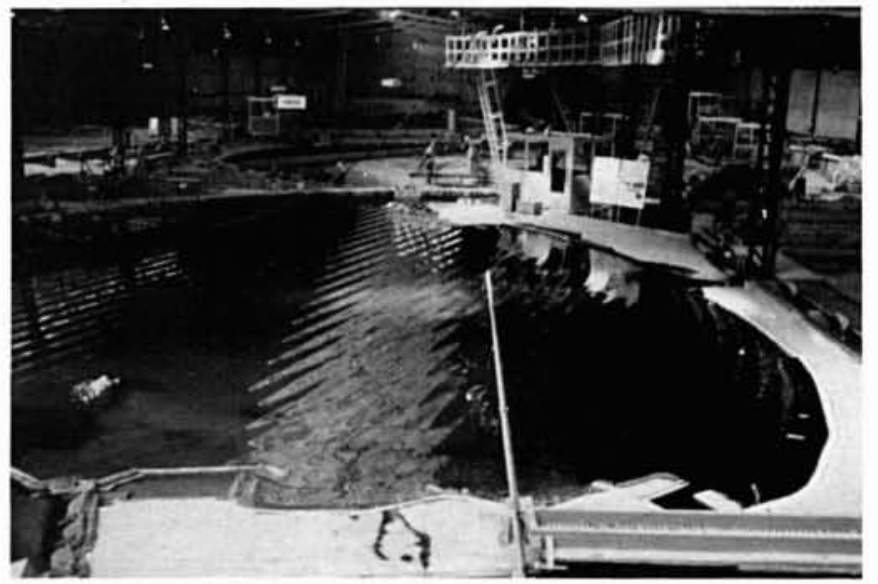

5.

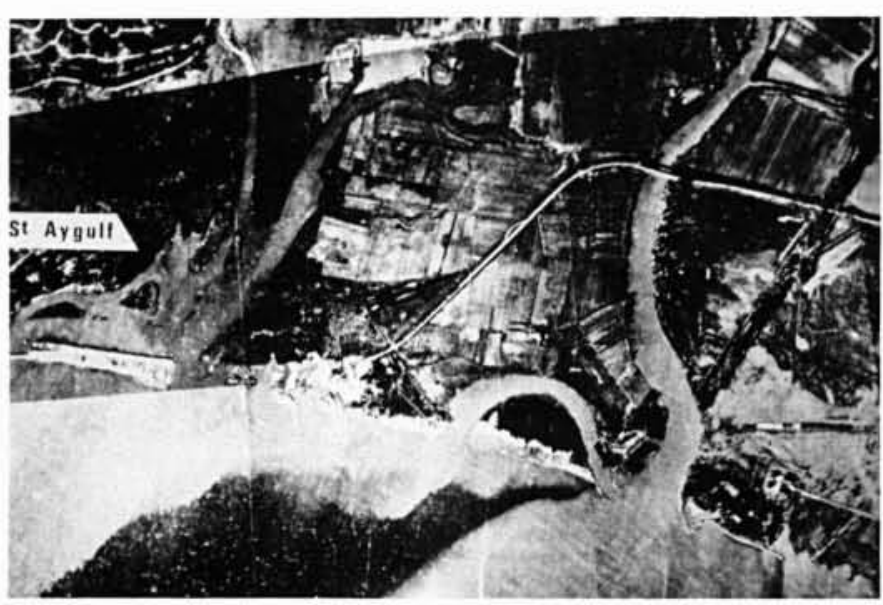

3.

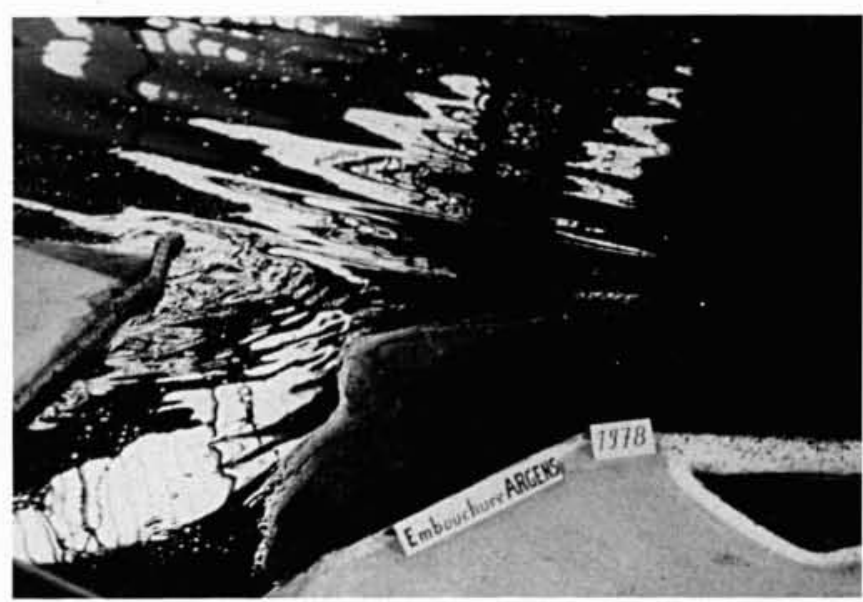

6.

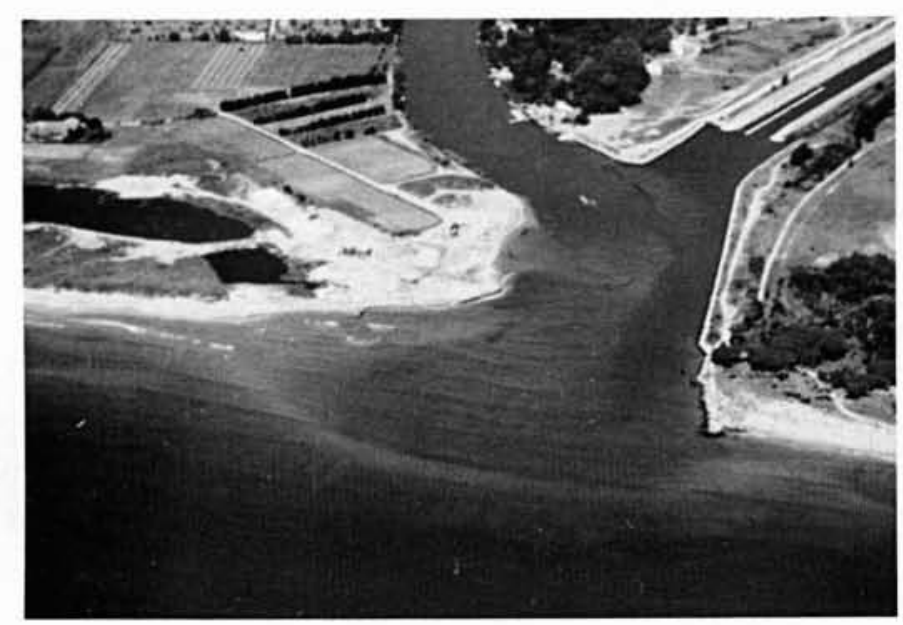

4.

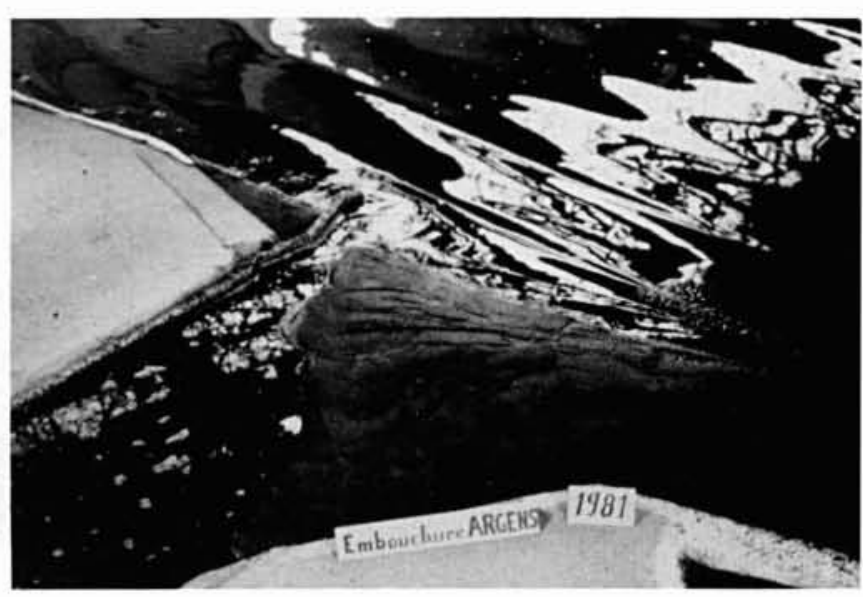

7. 


\section{Causes et importance des dégradations}

En 1980, la plage de Saint-Aygulf avait pratiquement disparu sur plus de un kilomètre (fig. 2), le recul moyen constaté étant évalué à $125 \mathrm{~m}$ depuis 1953 et la route littorale étant menacée.

Les causes de cette érosion massive ont quatre origines :

- la rupture du barrage de Malpasset en 1959 qui a engendré une lame d'eau considérable détruisant les installations riveraines et emportant près de $500000 \mathrm{~m}^{3}$ de sable vers le large, soit $100 \mathrm{~m}^{3}$ par mètre linéaire de plage (fig. 3) :

- l'action des houles qui entraine d'une part des mouvements dans le profil, avec une déperdition d'une partie des sédiments au cours des tempêtes, d'autre part un transit littoral du Sud vers le Nord estimé à $7000 \mathrm{~m}^{3}$ par an entre Saint-Aygulf et l'embouchure de l'Argens, au centre du littoral, et à 10000 à $15000 \mathrm{~m}^{3}$ par an entre l'Argens et Saint-Raphäl ;

- les extractions massives de sable qui ont eu lieu dans l'embouchure de l'Argens entre 1962 et 1978, à un taux supérieur aux apports fluviatiles $\left(20000 \mathrm{~m}^{3}\right.$ par an), et qui ont participé au déficit sédimentaire du littoral en créant un appel des matériaux vers l'intérieur de l'embouchure avec basculement du trait de côte sans possibilité de reprise par les houles d'Est (fig. 4):

- des réflexions de la houle sur les ouvrages de protection de la route nationale qui ont pu accentuer localement les phénomènes d'érosion.

\section{Les études effectuées}

Devant cet état préoccupant du littoral de Saint-Aygulf et compte tenu des projets de développement nautique et balnéaire de la plage de Fréjus située au Nord-Est de l'embouchure de l'Argens, la Municipalité de Fréjus a confié en 1980 une étude en nature et sur modèle réduit physique sédimentologique au Laboratoire central d'hydraulique de France (LCHF) actuellement regroupé avec la Sogreah.

\section{Caractéristiques du modèle réduit}

Ces études ont tout d'abord permis de préciser les causes exactes des dégradations associées aux conditions océanographiques, hydrologiques et sédimentologiques.

Un modèle réduit réalisé à une échelle en plan du $1 / 225^{\mathrm{e}}$ et en hauteur du $1 / 80^{\circ}$ permettait de représenter l'ensemble des phénomènes hydrauliques (houles, courants, surélévation du niveau des eaux) et les mouvements sédimentaires connus en nature en schématisant les sables par des particules plus légères de granulométrie appropriée (fig. 5).

Un réglage de ce modèle a permis de reproduire avec exactitude les évolutions des fonds avec un net recul de la plage de Saint-Aygulf et une certaine stabilité de la plage de Fréjus, ainsi que la disparition de la flèche à l'embouchure de l'Argens pendant les périodes d'extraction des granulats (fig. 6) et sa reconstitution après l'arrêt des dragages (fig. 7).

\section{Aménagements étudiés - Recherche d'une solution}

Sur ce modèle, on a étudié simultanément différentes solutions d'aménagements de la plage de Saint-Aygulf, de protection de l'embouchure de l'Argens, d'extension du port de plaisance de Saint-Aygulf et d'un nouveau port à Fréjus intégré dans la grande plage balnéaire avec une liaison avec l'ancien port Romain situé au centre de la ville (fig. 8).

Un premier essai a permis de confimer que la plage de Saint-Aygulf continuera de s'éroder si l'on n'intervient pas rapidement. Après quelques années, cette plage aura pratiquement disparu tandis que la plage de Fréjus se maintiendra.

Un deuxième essai, avec comme simple intervention un rechargement de la plage avec $75000 \mathrm{~m}^{3}$ de sable de même granulométrie que le sable existant, la réalisation d'un épi sur la rive droite de l'Argens et la mise en place d'un port de plaisance à Fréjus, montre qu'un simple rechargement de sable est insuffisant pour stabiliser la plage de SaintAygulf qui continue de s'éroder. Par contre, la digue à l'embouchure de l'Argens redresse le trait de côte dans ce secteur (fig. 9) et le port de plaisance de Fréjus permet, en bloquant le transit, d'élargir la plage située au Sud.

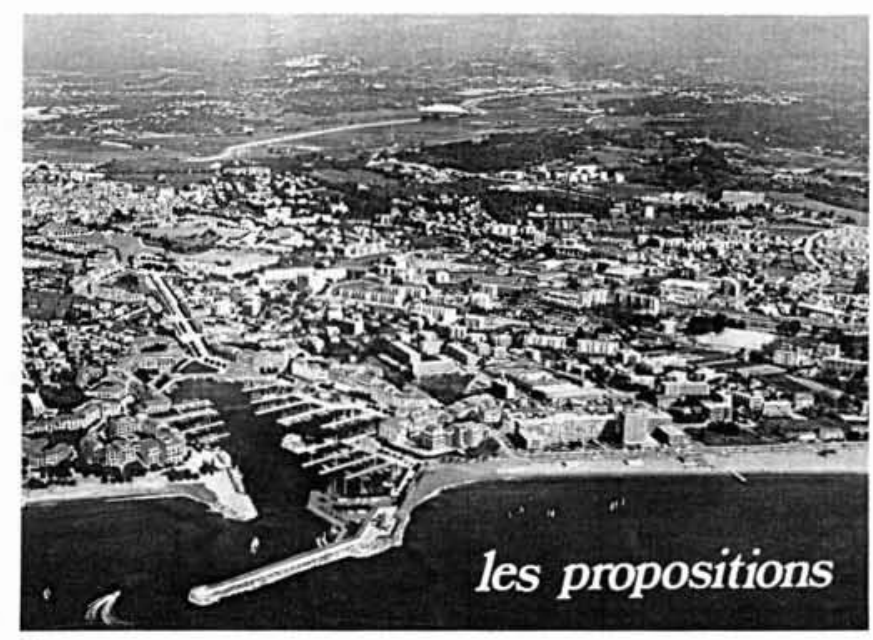

8. Projet du port de plaisance à Fréjus.

Après les études sur modèle réduit l'accès au port a été modifié.

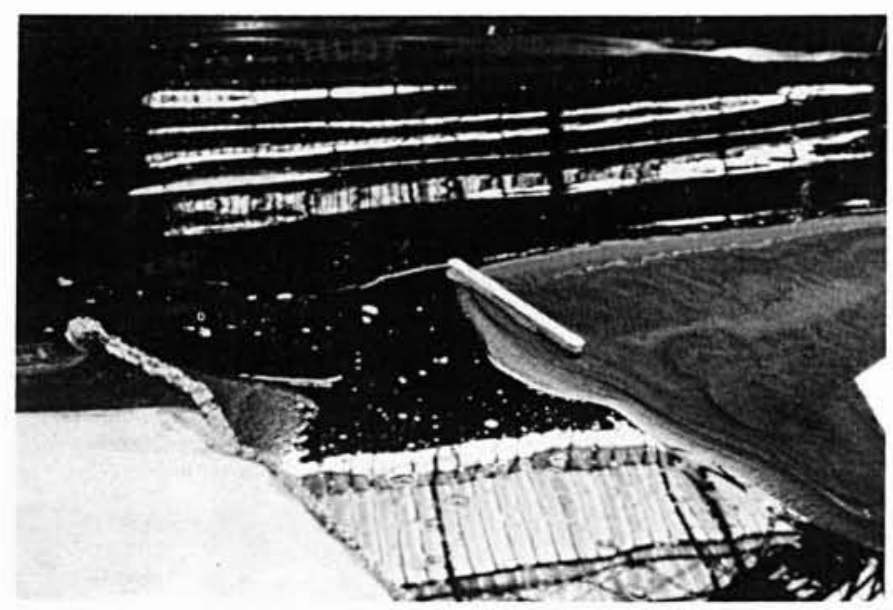

9. Protection de l'embouchure de l'Argens par un épi rive droite. 


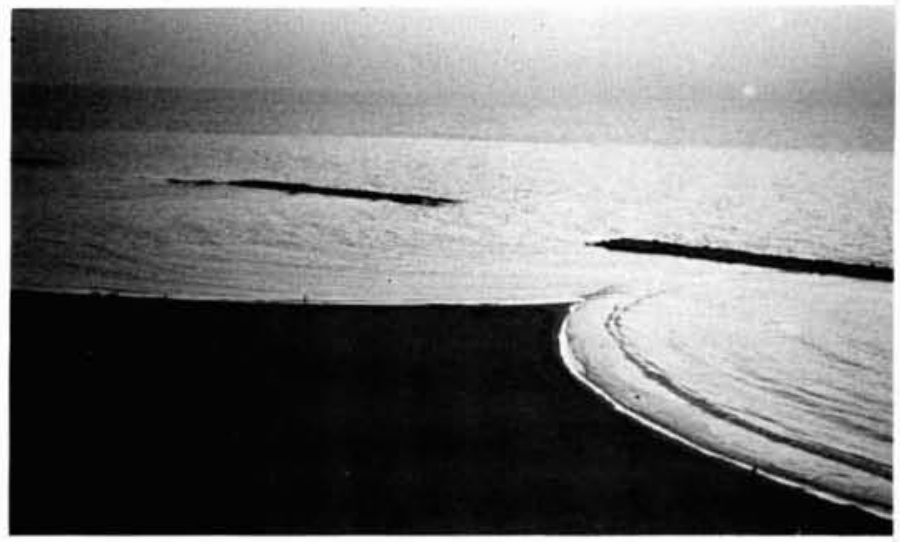

10.

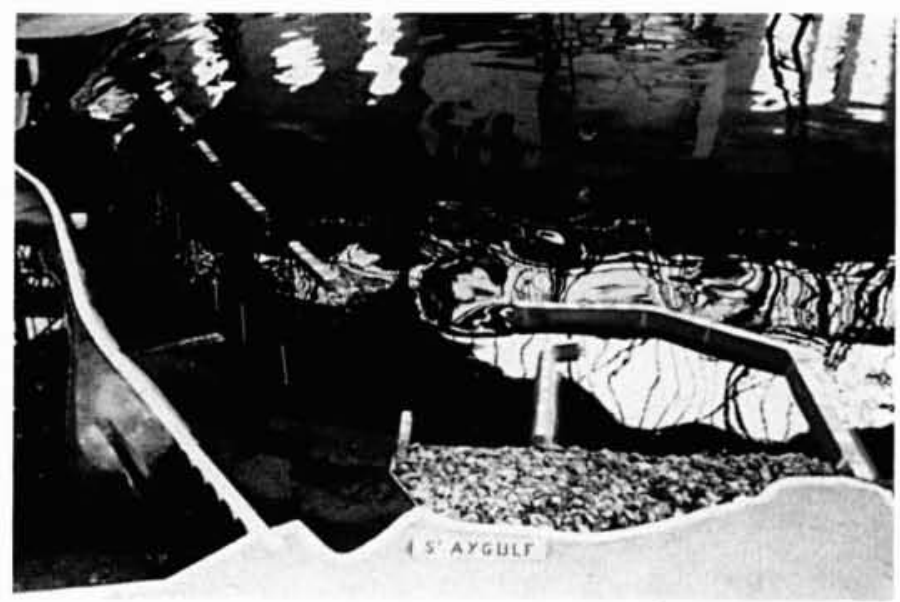

11.

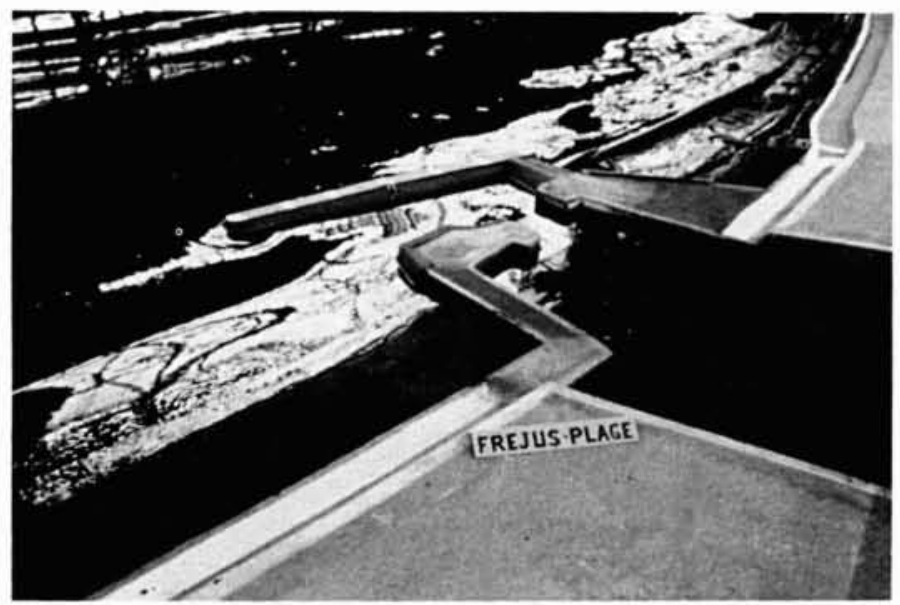

12.

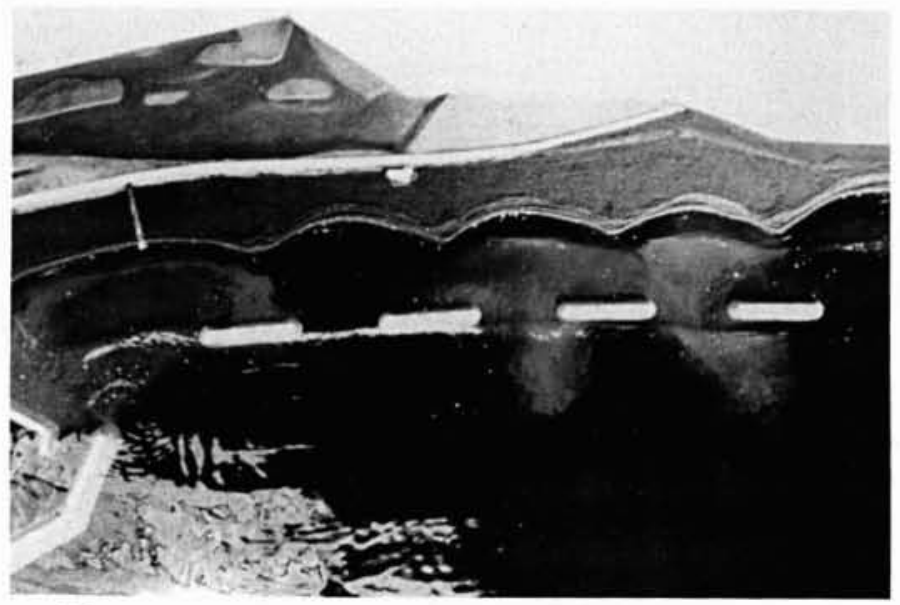

13.
10. Fonctionnement d'un brise-lames.

11. Etude des brise-lames devant la plage de Saint-Aygulf.

12. Etude de l'implantation du port de Fréjus.

13. Etude du renouvellement des eaux à l'intérieur des alvéoles.

Un troisième essai consistait à mettre au point une solution de protection de la plage de Saint-Aygulf à l'aide de brise-lames disposés en série et espacés de telle sorte qu'aucune concentration d'énergie ne puisse se produire entre eux. Ces brise-lames permettaient, en réduisant l'agitation dans leur zone d'ombre, de provoquer des dépôts de sédiments et d'éviter que les plages ne soient attaquées en tempêtes avec entraînement des sédiments vers le large (fig. 10).

Différents essais ont permis d'affiner et d'optimiser cette solution de protection en l'associant à des rechargements de sables plus ou moins importants et différentes implantations des ports de plaisance de Saint-Aygulf et de Fréjus.

\section{Solution préconisée}

La solution retenue a été expérimentée sur une longue période d'une dizaine d'années comportant (fig. 11 et 12) :

- quatre brise-lames de $120 \mathrm{~m}$ de long, espacés de $80 \mathrm{~m}$, implantés à $170 \mathrm{~m}$ des enrochements qui bordent la route nationale de Saint-Aygulf ;

- un rechargement de $200000 \mathrm{~m}^{3}$ de sable prélevé dans le site du futur port de Fréjus ;

- une suppression des extractions à l'embouchure de l'Argens ;

- une extension du port de Saint-Aygulf avec création d'une base nautique près de la plage de la Galiote ;

- un port de plaisance sur la plage de Fréjus avec une passe d'entrée s'ouvrant au Nord à une distance appropriée du littoral pour éviter son ensablement;

- un remblaiement de $80000 \mathrm{~m}^{3}$ de sable sur la plage de Fréjus permettant d'augmenter ses possibilités d'accueil balnéaire.

Cette solution retenue permettait d'obtenir une plage au contour ondulé, avec une avancée du trait de côte (tombolos) au droit des brise-lames et une largeur suffisamment importante pour répondre aux besoins balnéaires que l'attrait touristique de cette région nécessite.

Les ports de Saint-Aygulf et de Fréjus répondaient aux impératifs de sécurité nécessaires pour les bateaux de plaisance avec des risques d'ensablement aussi réduits que possible compte tenu de la position de ces installations près du rivage.

\section{Lutte contre la pollution - Renouvellement des eaux}

Précisons que l'implantation des brise-lames a été réalisée non seulement dans un souci de reconstituer les plages et d'assurer une protection suffisante contre l'érosion, mais 


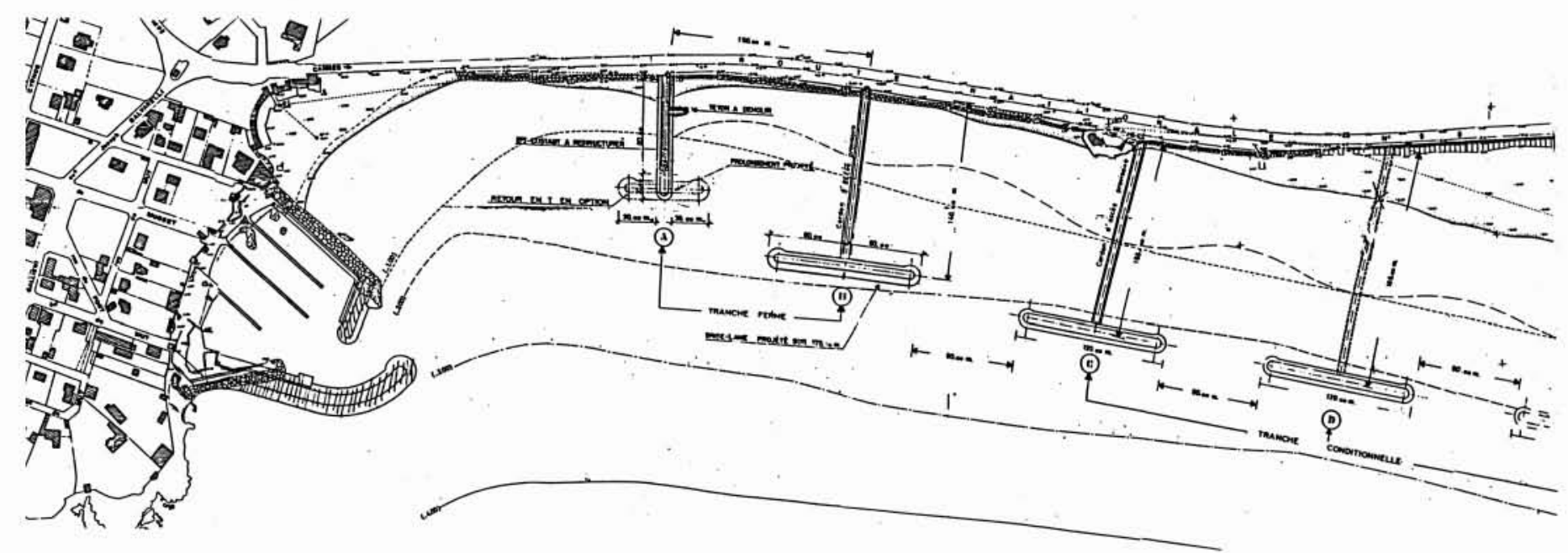

14. Implantation en nature des ouvrages retenus.

en tenant compte des possibilités de renouvellement des eaux à l'intérieur des alvéoles évitant ainsi une pollution des eaux et des sédiments dans les zones abritées (fig. 13).

\section{Les travaux réalisés}

En 1987, la commune de Fréjus adoptait les dispositions ainsi proposées et confiait à la Direction Départementale de l'Equipement, Service Maritime, l'étude et la conduite des travaux correspondant.

Le Conseil Municipal en adoptait le projet en juillet de la même année. Ce projet était conforme à celui retenu au cours des essais en modèle réduit (fig. 14).

\section{Caractéristiques des brise-lames}

L'émergence des brise-lames a été fixée à 2,10 m (cartes marines) soit à $1,80 \mathrm{~m}$ au-dessus du niveau moyen des eaux.

Les fonds avoisinent $-2,50 \mathrm{CM}$ à l'emplacement des ouvrages.

Le poids des enrochements de protection a été évalué par la formule de Hudson. Les enrochements du glacis supérieur des brise-lames étant plus lourds pour résister au laminage des lames déferlantes auxquelles de tels ouvrages submersibles sont exposés (fig. 15).

Le choix du mode de réalisation de la carapace selon une pose régulière en mosaïque plutôt qu'en vrac a été fait bien qu'il générera des franchissements un peu plus importants des ouvrages. Cette conception permet d'assurer une meilleure stabilité des brise-lames et améliore l'esthétique de ceux-ci.

Les ouvrages ont été construits depuis la terre, une rampe d'accès ayant été constituée en tout-venant pour le passage des engins de chantier. Une réalisation par voie maritime eut été beaucoup trop onéreuse.
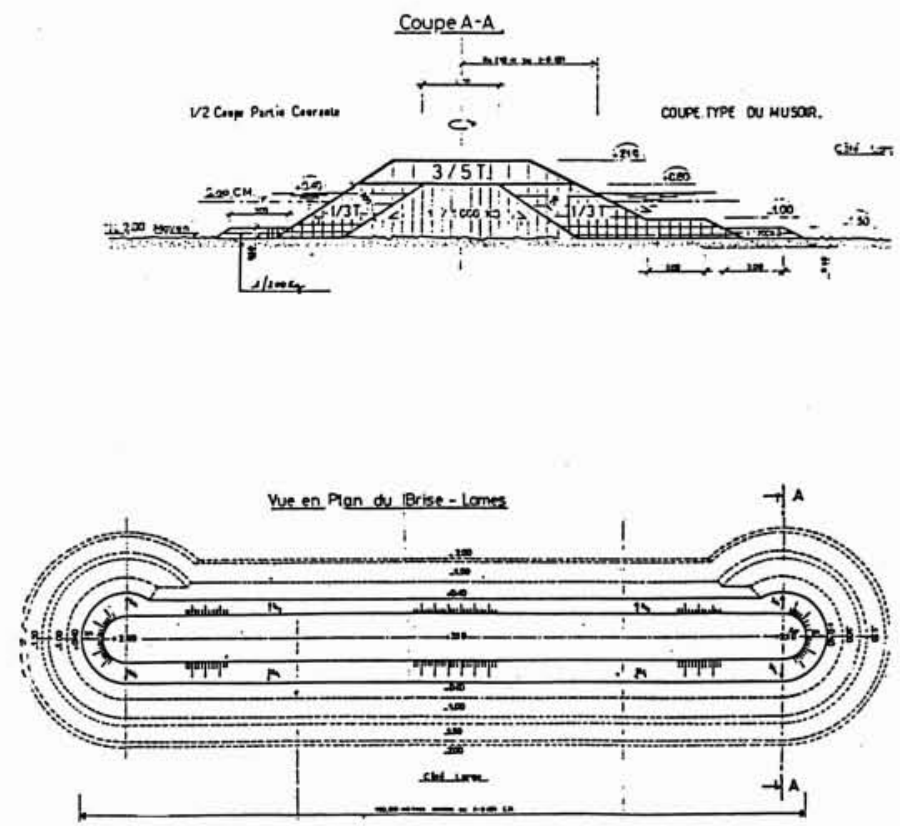

15. Coupe des brise-lames.

\section{Importance des travaux}

Les travaux ont été confiés, après appel d'offres, à l'entreprise Société Travaux, Constructions, Matériaux (S.T.C.M.) de Fréjus.

Ils ont nécessité l'apport de :

- plus de 50000 tonnes de tout-venant de carrière pour confectionner des pistes d'accès, assise et noyau des briselames dont 10000 tonnes des premiers cordons d'accès ont été récupérés pour les suivants; 


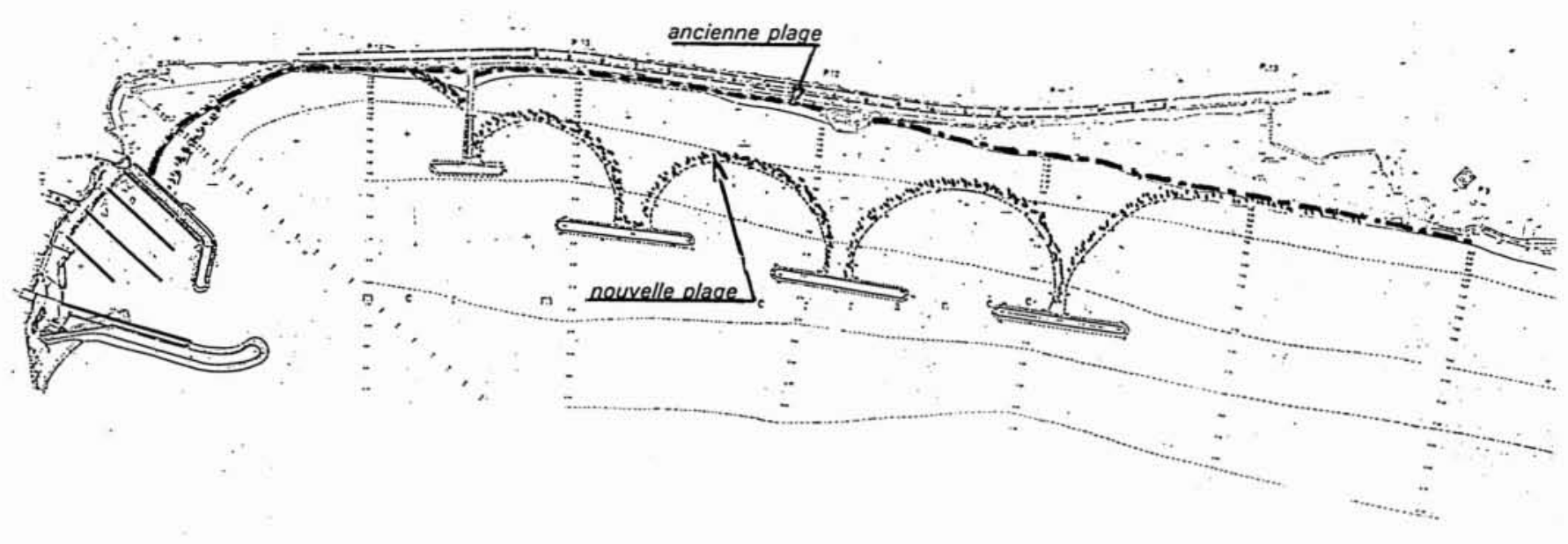

16. Comparaison du tracé de l'ancienne et de la nouvelle plage de Saint-Aygulf.

— plus de 40000 tonnes d'enrochements de 1 à 5 tonnes. Le coût de ces travaux a été de 10 millions de francs. La commune de Fréjus a obtenu, pour contribuer au financement de ces travaux :

- des subventions notamment du Conseil Général du Var, de la Région et de l'Etat,

- une participation de la SEM de l'Aire Fréjussienne (SEMAF) concessionnaire de Port Fréjus dans le cadre des charges de la ZAC du port.

Ces ouvrages ont été réalisés dans le cadre d'une concession d'endigage et d'utilisation des dépendances hors port du domaine public maritime (Décret $n^{\circ} 79-518$ du 29 juin 1979) consentie par l'Etat à la commune de Fréjus.

Les travaux ont été effectués principalement au cours du second semestre 1988 et du premier semestre 1989.

\section{Rechargement de la plage}

La municipalité de Fréjus a programmé la réalisation des brise-lames de telle sorte qu'ils soient achevés lors du dragage du bassin de Port Fréjus.

En effet, le rechargement des plages de Fréjus constituait l'essentiel des mesures compensatoires à la création de ce port intérieur.

D'autre part, les sondages géologiques effectués dans le cadre des études préliminaires de ce projet portuaire, avaient mis en évidence les qualité et quantité de sable à draguer pour le creusement du bassin.

C'est ainsi que plus de $200000 \mathrm{~m}^{3}$ de sédiments sableux de granulométrie semblable à celle du sable en place ont été transférés sur la plage de Saint-Aygulf.

Afin de ne pas perturber la circulation routière dans ce secteur, ce transfert a été effectué grâce à la mise en place d'un pipeline de $4000 \mathrm{~m}$ de longueur.

La barge refoulait dans ce sabloduc de $40 \mathrm{~cm}$ de diamètre, un mélange d'eau de mer et de sable (10 à $40 \%$ maximum) à une vitesse de $4,50 \mathrm{~m} / \mathrm{s}(16 \mathrm{~km} / \mathrm{h})$ aidée en cela par 3 pompes-relais.

Fonctionnant jour et nuit, y compris le week-end (hors opérations de maintenance), cette installation assurait un débit moyen de $1000 \mathrm{~m}^{3}$ de sable/jour.
Cet apport de sable a été réalisé par l'entreprise Tournaud, 69 Irigny, au cours des mois du $2^{\mathrm{e}}$ trimestre 1989.

Le coût de ce rechargement a été totalement pris en charge par la SEMAF.

\section{Résultats des travaux - Perspectives d'avenir}

L'ensemble des travaux réalisés le long du littoral de SaintAygulf a permis de recréer une plage dans ce secteur particulièrement menacé. Parallèlement, le petit port de plaisance de Saint-Aygulf était agrandi et celui de Fréjus entrepris.

La superficie de plage reconstituée à Saint-Aygulf entre l'ancien rivage tel que délimité par Décret du 25 février 1983 et le rivage actuel, dépasse 6 hectares, le tracé de la nouvelle plage étant donné sur les figures 16 et 17.

Cette nouvelle plage sera aménagée dans un premier temps par des installations " légères » comportant le déplacement de deux établissements existants, des postes de secours et des équipements sanitaires, un cheminement piétonnier, un parking longitudinal en bordure de la RN 98 et un traitement paysager.

Parallèlement, il sera procédé à une réflexion très globale en vue d'un aménagement durable de ce secteur qui, avec les Etangs de Villepey, constitue un patrimoine exceptionnel à sauvegarder (fig. 18).

Ainsi, partant d'un littoral entièrement dégradé par une accumulation de phénomènes naturels et humains qui ont contribué à la disparition de la plage de Saint-Aygulf, les travaux entrepris au cours des dernières années ont permis une reconquête de la plage sur la mer.

S'appuyant sur un diagnostic précis en nature et sur des études minutieuses à l'aide d'un modèle réduit physique sédimentologique permettant d'éviter les aléas et les incertitudes des travaux à la mer, les résultats obtenus après réalisation des ouvrages confirment les prévisions et apportent à la région de Saint-Aygulf et de Fréjus un accroissement de son potentiel nautique et balnéaire particulièrement bien adapté à sa vocation touristique et à son environnement. 
PROTECTION DU LITTORAL : LA PLAGE DE SAINT-AYGULF

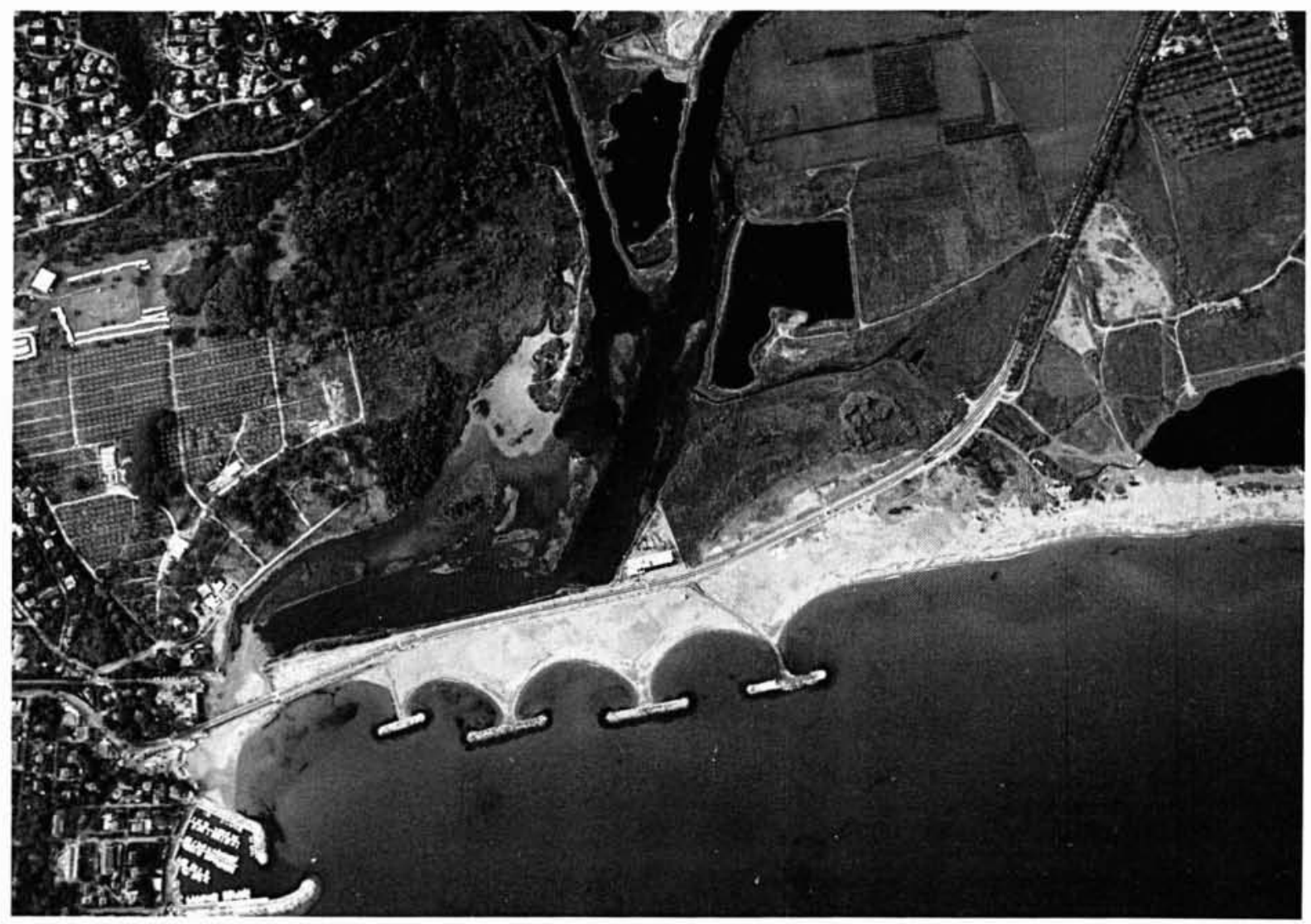

17. Vue aérienne de la nouvelle plage de Saint-Aygulf.

18. Le littoral aménagé de Saint-Aygulf et les Etangs de Villepey.

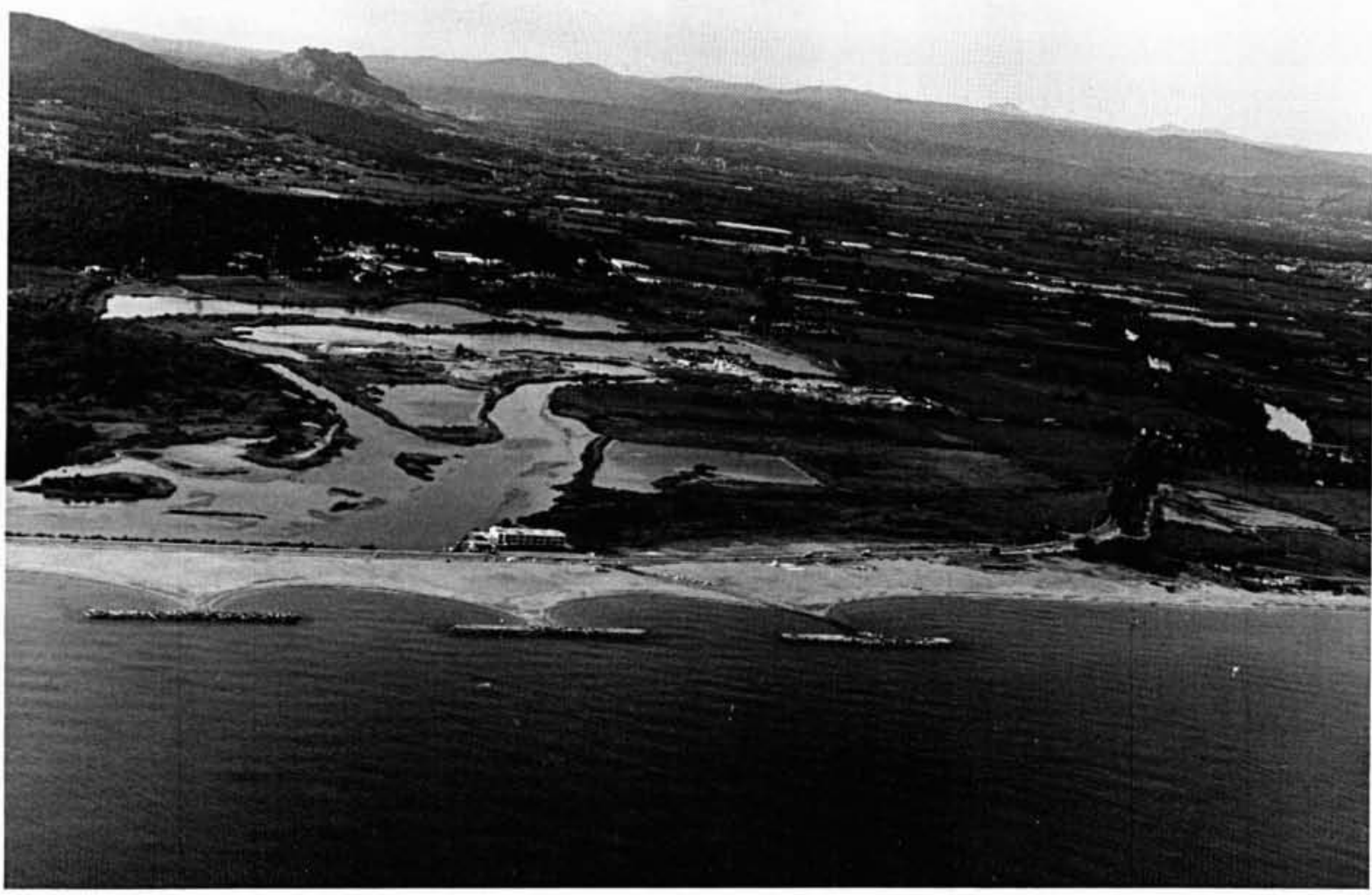

